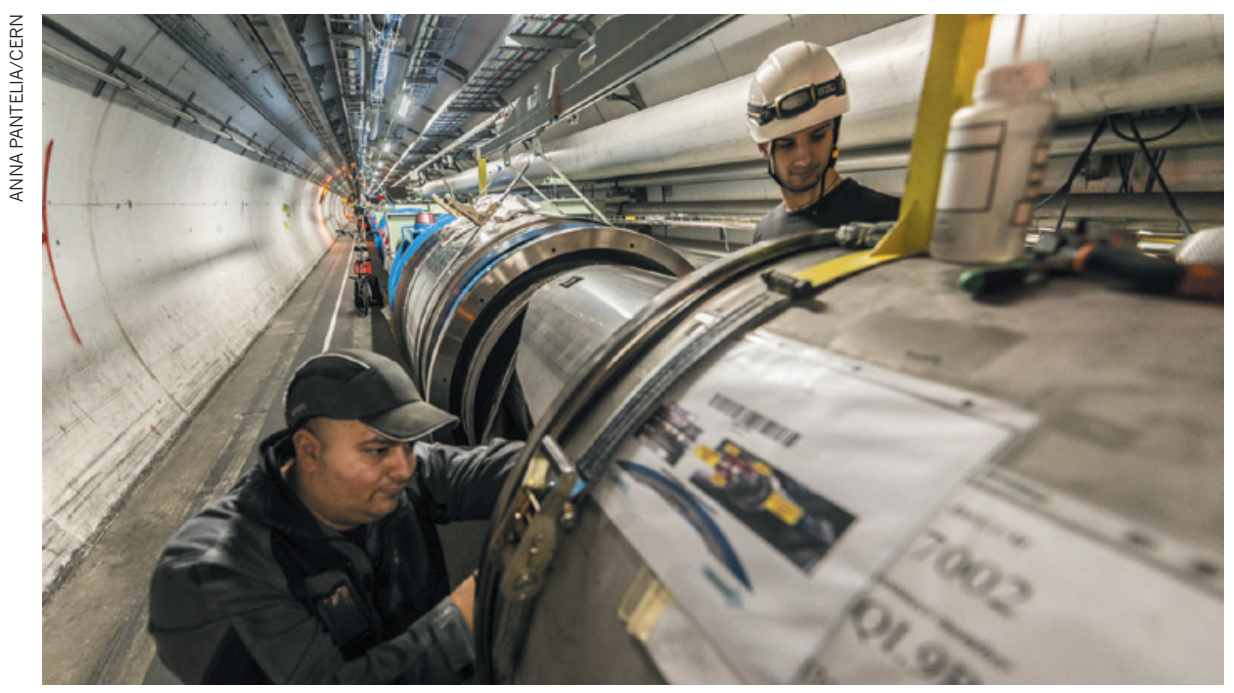

Discoveries at the Large Hadron Collider, now being upgraded, will inform designs for the next accelerator.

\title{
PARTICLE PHYSICS
}

\section{Physicists plan to build a bigger LHC}

\section{Accelerator ring would be 100 kilometres around and run at seven times the energy of the Large Hadron Collider.}

\section{BY EUGENIE SAMUEL REICH}

$\mathrm{W}$ hen Europe's Large Hadron Collider (LHC) started up in 2008, particle physicists would not have dreamt of asking for something bigger until they got their US\$5-billion machine to work. But with the 2012 discovery of the Higgs boson, the LHC has fulfilled its original promise - and physicists are beginning to get excited about designing a machine that might one day succeed it: the Very Large Hadron Collider (VLHC).

"It's only prudent to try to sketch a vision decades into the future," says Michael Peskin, a theoretical physicist at SLAC National Accelerator Laboratory in Menlo Park, California, who presented the VLHC concept to a US government advisory panel on 2 November.

The giant machine would dwarf all of its predecessors (see 'Lord of the rings'). It would collide protons at energies around 100 teraelectronvolts $(\mathrm{TeV})$, compared with the planned $14 \mathrm{TeV}$ of the LHC at CERN, Europe's particle-physics lab near Geneva in Switzerland. And it would require a tunnel 80-100 kilometres around, compared with the LHC's $27-\mathrm{km}$ circumference. For the past decade or so, there has been little research money available worldwide to develop the concept. But this summer, at the Snowmass meeting in
Minneapolis, Minnesota - where hundreds of particle physicists assembled to dream up machines for their field's long-term future the VLHC concept stood out as a favourite.

Some physicists caution that the VLHC would be only a small part of the global particle-physics agenda. Other priorities include: upgrading the LHC, which shut down in February for two years to boost its energies from $7 \mathrm{TeV}$ to $14 \mathrm{TeV}$; plans to build an International Linear Collider in Japan, to collide beams of electrons and positrons as a complement to the LHC's proton findings; and a major US project to exploit high-intensity neutrino beams generated at the Fermi National Accelerator

\section{LORD OF THE RINGS}

Physicists are discussing a proton-colliding machine that would dwarf the energy of its predecessors.

Very Large Hadron Collider (suggested)

100 km

$100 \mathrm{TeV}^{*}$

Large Hadron Collider

27 km

$14 \mathrm{TeV}$

Tevatron (closed)

Circumference: $6.3 \mathrm{~km}$

Energy: 2 TeV

*TeV, teraelectronvolt.
Laboratory in Batavia, Illinois. Jonathan Rosner, a particle physicist at the University of Chicago, Illinois, who convened Snowmass, says that these forthcoming projects should be the focus. "It's premature to highlight the VLHC," he says.

In some ways, the interest in the VLHC is a sign that particle physicists are returning to their roots, pushing to ever higher energies to find the fundamental building blocks of nature.

They will have to justify it, however. The discovery of the Higgs particle lends support to the idea that some particles have mass because they interact with a pervasive, treacle-like Higgs field. Yet many aspects of the discovery are still not understood, including why the mass of the Higgs particle is so large. One way of explaining its heaviness is through supersymmetry theory, in which known particles are coupled with heavier ones that might be observed in bigger particle colliders. Although the LHC has not detected any signs of supersymmetry, Peskin hopes that a hint may come before the end of the decade, which would help to inform the design of a larger machine.

One advocate of a bigger machine is Nima Arkani-Hamed, a theoretical physicist at the Institute for Advanced Study in Princeton New Jersey. In December, he will help to launch an institute in Beijing called the Center for Future High Energy Physics. Part of its explicit mission, he says, is to explore the physics that a future proton collider might investigate. William Barletta, an accelerator physicist at the Massachusetts Institute of Technology in Cambridge, says that this work is crucial to identify a machine size that will maximize the science per dollar. "We won't just give handwaving arguments," he says.

To build a 100-TeV machine, Barletta adds, physicists will need to develop superconducting magnets that can operate at higher fields than the current generation, perhaps 20 tesla instead of 14 tesla. One leading candidate material for such magnets is niobium tin, which can withstand higher fields but is expensive and must be cooled below 18 kelvin.

CERN is developing its own plans for a collider that is similar to the VLHC. CERN accelerator physicist Michael Benedikt is leading a study of a 'very high energy large hadron collider' that would pass under Lake Geneva. It would have the same key parameters as the suggested VLHC: a circumference of $80-100 \mathrm{~km}$ and a collision energy of $100 \mathrm{TeV}$. Benedikt suggests that construction might begin in the 2020s so that the machine could be completed soon after the LHC shuts down for good around 2035. "One would not want to end up with a huge gap for highenergy physics," he says. He adds that it is too early to offer a price tag. But other physicists speculate that a next-generation collider would have to cost less than $\$ 10$ billion for the project to be politically plausible. 